\title{
A high-protein formula increases colonic peptide transporter 1 activity during neonatal life in low-birth-weight piglets and disturbs barrier function later in life
}

\author{
Gaëlle Boudry*, Véronique Rome, Cécile Perrier, Agnès Jamin, Gérard Savary and \\ Isabelle Le Huerou-Luron \\ INRA UR1341 ADNC, Domaine de la Prise, 35590 Saint-Gilles, France
}

(Submitted 19 December 2013 - Final revision received 12 May 2014 - Accepted 14 May 2014 - First published online 14 August 2014)

\section{Abstract}

Dietary peptides are absorbed along the intestine through peptide transporter 1 (PepT-1) which is highly responsive to dietary protein level. PepT-1 is also involved in gut homeostasis, both initiating and resolving inflammation. Low-birth-weight (LBW) neonates are routinely fed a high-protein (HP) formula to enhance growth. However, the influence of this nutritional practice on PepT-1 activity is unknown. Intestinal PepT-1 activity was compared in normal-birth-weight (NBW) and LBW piglets. The effect of HP $v$. normal-protein (NP) formula feeding on PepT-1 activity and gut homeostasis in LBW piglets was evaluated, during the neonatal period and in adulthood. Flux of cephalexin (CFX) across the tissue mounted in Ussing chambers was used as an indicator of PepT-1 activity. CFX flux was greater in the ileum, but not jejunum or colon, of LBW than NBW piglets during the neonatal period. When LBW piglets were formula-fed, the HP formula increased colonic CFX during the 1st week of life. Later in life, intestinal CFX fluxes and barrier function were similar whether LBW pigs had been fed NP or HP formula. However, colonic permeability of HP- but not NP-fed pigs increased when luminal pH was brought to 6.0. The formyl peptide $N$-formyl methionyl-leucyl-phenylalanine conferred colonic barrier protection in HP-fed piglets. Heat shock protein 27 levels in the colonic mucosa of HP-fed LBW pigs correlated with the magnitude of response to the acidic challenge. In conclusion, feeding a HP formula enhanced colonic PepT-1 activity in LBW pig neonates and increased sensitivity of the colon to luminal stress in adulthood.

Key words: Peptide absorption: Low-birth-weight neonates: $N$-Formyl methionyl-leucyl-phenylalanine: Formulas

The predominant mode of intestinal absorption of protein hydrolysis products in adults and infants is through $\mathrm{H}^{+}$coupled peptide transporter 1 (PepT-1) ${ }^{(1)}$. It allows absorption of a large variety of di- and tripeptides at the apical membrane that are further hydrolysed by peptidases in the cytoplasm of enterocytes. PepT-1 expression (mRNA and/or protein) increases during the fetal period to peak either at birth or between postnatal day (PND) 3 and 5 in rats and piglets. It then declines with age in both species ${ }^{(2-5)}$. An original feature of PepT-1 development is its relatively high expression in the proximal colon of neonatal rats and piglets during the very early life period, i.e. PND2 or PND $3^{(2,4)}$. PepT-1 expression and activity in adults are responsive to changes in the quantity and composition of the substrate, showing greater activity when the availability of protein or di- and tripeptides is increased $^{(1,3)}$. This regulation by dietary substrate appears to occur by increasing the gene transcription rate and/or mRNA stability $^{(6)}$. Human low-birth-weight (LBW) neonates are usually fed high-protein (HP) formulas to ensure rapid postnatal catch-up growth ${ }^{(7,8)}$. Interestingly, the postnatal development of PepT-1 mRNA in LBW piglets differs from that of normal-birth-weight (NBW) piglets, with significantly greater levels of PepT-1 mRNA in the ileum and proximal colon of LBW compared with NBW piglets at PND2 ${ }^{(4)}$. However, it remains to be known whether feeding a HP formula modifies PepT-1 activity in the neonatal period, especially in LBW neonates.

Besides its role as peptide transporter, a body of literature has emerged on the role of PepT-1 in gut homeostasis. Indeed, in addition to dietary peptides, PepT-1 is able to transport bacterial peptides such as $n$-formyl and muramyl peptides present in gut luminal content, which are potent

Abbreviations: CFX, cephalexin; FD-4, fluorescein isothiocyanate-dextran 4000; f-MLP, $N$-formyl methionyl-leucyl-phenylalanine; HP, high protein; Hsp, heat shock protein; LBW, low birth weight; MES, 2-(N-morpholino)ethane sulfonic acid; NBW, normal birth weight; NP, normal protein; PepT-1, peptide transporter 1 ; PND, postnatal day.

*Corresponding author: Dr G. Boudry, fax +33 2234850 80, email gaelle.boudry@rennes.inra.fr 
neutrophil chemotactic substances ${ }^{(9)}$. PepT-1 was therefore believed to promote inflammation. As such, it has been reported that PepT-1 is highly expressed in a chronically inflamed colon in adults ${ }^{(10)}$. Infusion of the model peptide $N$-formyl methionyl-leucyl-phenylalanine (f-MLP) elicits neutrophil infiltration and inflammation in the jejunum of adult rats within $4 \mathrm{~h}$. Inhibition of PepT-1 reduced this effect ${ }^{(11)}$. Infusion of $\mathrm{f}$-MLP also increases ileal permeability in rats through a neutrophil-derived oxidantdependent mechanism ${ }^{(12)}$. However, participation of formyl peptide receptors 1 and 2 in gut homeostasis and repair after injury ${ }^{(13)}$ has also been demonstrated. Cell protection mechanisms against oxidative and inflammatory damage by f-MLP through heat shock protein (Hsp) 27 induction $^{(14)}$ seem to be involved in this protective effect.

Recent data indicated that changes in gut homeostasis during early life have a durable impact on gut function. This was first exemplified by early life stress that disturbs the cross-talk between gut microbiota, barrier function and neurons in rat neonates and is followed by greater response to gut inflammation or visceral hypersensitivity in adults ${ }^{(15)}$. Our group recently demonstrated that HP formula feeding influences distal intestinal homeostasis in pigs both during the neonatal period and in adulthood ${ }^{(16,17)}$. Based on these literature data and our published data on the developmental pattern of PepT-1 mRNA in LBW piglets $^{(4)}$, we hypothesised that LBW piglets have increased PepT-1 activity in the distal intestine that might be accentuated by HP formula feeding. Furthermore, we speculated that changes in PepT-1 activity would have long-term consequences on distal intestine barrier function and homeostasis. The objectives of the present study were therefore (1) to investigate PepT-1 activity in LBW and NBW piglets at different ages, (2) to examine the effect of HP formula feeding on PepT-1 activity in LBW piglets and (3) to evaluate whether distal intestine barrier function is disturbed in adult LBW pigs fed a HP formula during neonatal life.

\section{Materials and methods}

\section{Animal procedures}

The experimental protocol was designed in compliance with the recommendations of the French and European law (Décret: 2001-464 29/05/01, 86/609/CEE) for the care and use of laboratory animals under the certificate of authorisation to experiment on living animals no. 35-69.

Cross-bred (Piétrain $\times($ Large White $\times$ Landrace $)$ ) piglets from the experimental herd of INRA (St-Gilles, France) were used in two separate experiments.

Expt 1: comparison of peptide transporter 1 activity in low-birth-weight and normal-birth-weight suckling piglets. A total of fifteen LBW piglets (average birth weight $1.01 \mathrm{~kg}$, range $0.74-1.21 \mathrm{~kg}$ ) and fifteen NBW piglets (average birth weight $1.5 \mathrm{~kg}$, range $1.32-1.64 \mathrm{~kg}$ ) were selected at birth based on birth weight. They originated from eight different litters representative of our herd (number of piglets per litter 15.5 (SEM 0.6) and average birth weight 1.24 (SEM 0.03$) \mathrm{kg}$ ). LBW piglets were chosen with a birth weight $30 \%$ lower than the average birth weight of the litter. Due to this constraint in birth weight, it was not possible to take sex into account. However, the number of same-sex and non-same sex pairs of piglets was balanced among the three different age groups. Piglets suckled their mother until PND21 and had no access to creep feed. Of the piglets, one-third was killed at PND2, one-third at PND7 and onethird at PND21. Piglets were anaesthetised with isoflurane delivered through a veterinary anaesthesia ventilator. They were then euthanised by intracardiac T-61 injection (Elvetis). After laparotomy, $15 \mathrm{~cm}$ segments of jejunum $(20 \mathrm{~cm}$ distal from the Treitz ligament), ileum $(20 \mathrm{~cm}$ proximal to the ileo-caecal junction) and colon $(15 \mathrm{~cm}$ distal from the ileocaecal junction) were collected, rinsed with cold $0.9 \% \mathrm{NaCl}$ solution and immediately placed in Ringer's bicarbonate (composition in mmol/l: $\mathrm{Na}^{+} 145, \mathrm{Cl}^{-} 128, \mathrm{PO}_{4}^{3-} 0 \cdot 32, \mathrm{Ca}^{2+} 2$, $\left.\mathrm{Mg}^{2+} 1, \mathrm{HCO}_{3}^{-} 25, \mathrm{SO}_{4}^{2-} 1, \mathrm{~K}^{+} 6 \cdot 3, \mathrm{pH} 7 \cdot 4\right)$ for the Ussing chamber experiment (cephalexin (CFX) flux experiment, without electrophysiological measurements).

Expt 2: effect of a high-protein formula on peptide transporter 1 activity and mRNA expression in low-birth-weight piglets. A total of forty-eight LBW piglets were fed from PND2 to PND28 a normal-protein (NP) formula $(50 \mathrm{~g}$ protein/l) or a HP formula (77 g protein/l; Table 1), and then a pig-dedicated diet until PND160 as described already ${ }^{(16)}$. Piglets were killed at PND7 ( $n 6$ per group), PND28 ( $n 6$ per group) and PND160 ( $n 12$ per group). They were euthanised by electrocution followed by exsanguination. After laparotomy, the segments of the distal ileum and proximal colon were collected as described above. Pieces of tissue were collected for the Ussing chamber experiment (CFX flux experiment, without electrophysiological measurements at PND7, PND28 and PND160 and barrier function and electrophysiological measurements at PND160). Pieces of tissue (100 mg) were also collected and either placed in RNAlater (Applied Biosystems) for $24 \mathrm{~h}$ at $4^{\circ} \mathrm{C}$ and stored at $-20^{\circ} \mathrm{C}$ until RNA extraction or snap-frozen in liquid $\mathrm{N}_{2}$ and stored at $-80^{\circ} \mathrm{C}$ until Western blot analysis.

\section{Ussing chamber experiment}

Intestinal tissues were stripped of the external muscle layers (except for colonic tissues at PND7 and PND28 where the muscle layers were left intact to avoid deteriorating the mucosa when stripping) and opened along the antimesenteric border and then mounted in the Ussing chamber (World Precision Instrument). The chamber opening exposed $0.67 \mathrm{~cm}^{2}$ of tissue surface area to $8 \mathrm{ml}$ of circulating oxygenated buffer (see below for composition) at $39^{\circ} \mathrm{C}$.

Table 1. Composition of the neonatal formula

\begin{tabular}{lccc}
\hline & NP & HP & Sow milk ${ }^{(36)}$ \\
\hline Composition & & & \\
$\quad$ Energy $(\mathrm{MJ} / \mathrm{l})$ & 4.75 & $5 \cdot 03$ & $4 \cdot 70$ \\
Protein $(\mathrm{g} / \mathrm{l})$ & 51 & 77 & 50 \\
Fat $(\mathrm{g} / \mathrm{l})$ & 82 & 79 & 80 \\
Carbohydrate $(\mathrm{g} / \mathrm{l})$ & 49 & 46 & 51 \\
\hline
\end{tabular}

$\mathrm{NP}$, normal protein; HP, high protein. 
PepT-1 activity in different segments of the intestine was evaluated by measuring mucosal-to-serosal fluxes of CFX (CFX, an aminocephalosporin transported through PepT-1 ${ }^{(18)}$ and not metabolised by the intestine ${ }^{(19)}$ ). One piece of proximal jejunal, distal ileal and proximal colonic tissue per piglet (PND2, PND7 and PND21 for Expt 1 and PND7, PND28 and PND160 for Expt 2) was bathed in $10 \mathrm{~mm}$ 2-(N-morpholino)ethane sulfonic acid (MES) Tris buffer (in mM: $\mathrm{NaCl} 137, \mathrm{KCl} 54, \mathrm{CaCl}_{2} 28, \mathrm{MgSO}_{4} 1, \mathrm{H}_{2} \mathrm{PO}_{4}$ 0.3, MES Tris 10, mannitol 10, $\mathrm{pH} 6 \cdot 0$ ) on the mucosal side and a HEPES Tris buffer (in mM: $\mathrm{NaCl} 137, \mathrm{KCl} 54, \mathrm{CaCl}_{2} 28$, $\mathrm{MgSO}_{4} 1, \mathrm{H}_{2} \mathrm{PO}_{4} 0 \cdot 3$, HEPES Tris 10 , glucose $10, \mathrm{pH} 7 \cdot 4$ ) on the serosal side. In preliminary experiments, we evaluated CFX passage across the intestinal tissues of NBW piglets at PND28 with different mucosal concentrations of CFX $(0-200 \mathrm{~mm})$. For all the intestinal segments studied, the flux of CFX across the mucosa plateaued, starting at a mucosal CFX concentration of $50 \mathrm{~mm}$, which was therefore chosen. CFX was added in the mucosal compartment of the Ussing chambers (World Precision Instrument) after a 20 min equilibration period. Serosal sample was collected after $60 \mathrm{~min}$ to measure CFX concentration by HPLC.

At PND160, we also evaluated ileal and colonic barrier function in standard or challenged conditions. Briefly, three adjacent pieces of ileum or colon per pig were mounted in Ussing chambers (World Precision Instrument). The transepithelial potential difference of the intestinal tissue was clamped at $0 \mathrm{mV}$ by an external current (short-circuit current, $I_{\mathrm{SC}}$ ). Transepithelial resistance $(R)$ was calculated from Ohm's law from current deflections in response to $3 \mathrm{mV}$ transepithelial voltage pulses of $300 \mathrm{~ms}$ every $10 \mathrm{~s}$. We used a mild acidic stress (change from pH $7 \cdot 4$ to 6.0) associated or not with the inflammatory peptide f-MLP. Acidic conditions up to $\mathrm{pH} 6.0$ (as opposed to $\mathrm{pH}$ 5.5) do not disturb intestinal epithelial cell barrier function (transepithelial resistance and flux of sulphonic acid) in normal conditions ${ }^{(20)}$, but enhances epithelial barrier sensitivity to inflammatory stimulus ${ }^{(21)}$. On both sides, one segment was mounted with Ringer buffer ( $\mathrm{pH} 7 \cdot 4$ ), supplemented with $10 \mathrm{~mm}$-mannitol and $10 \mathrm{~mm}-$ glucose on the luminal and serosal sides, respectively. Thereafter, two adjacent segments of tissues were mounted with 10 mm-MES Tris buffer ( $\mathrm{pH} \mathrm{6.0)}$ on the luminal side and 10 mM-HEPES Tris buffer ( $\mathrm{pH} 7 \cdot 4)$ on the serosal side. After a 20 min equilibration period, $100 \mathrm{~mm}-\mathrm{f}-\mathrm{MLP}$ was added to the mucosal side of one of these later chambers. Since f-MLP absorption through PepT-1 requires acidic conditions on the mucosal side, we omitted the $\mathrm{pH} 7 \cdot 4+\mathrm{f}-\mathrm{MLP}$ condition. After $10 \mathrm{~min}, 10 \mu \mathrm{m}$-horseradish peroxidase and fluorescein isothiocyanate-dextran 4000 (FD-4) were added to the luminal side in all chambers, and serosal samples were collected every $30 \mathrm{~min}$ for $120 \mathrm{~min}$. Concentrations of horseradish peroxidase and FD-4 were determined as described already ${ }^{(16)}$.

\section{Determination of cephalexin concentration}

The concentration of CFX in samples was determined by HPLC using a C18 column (AccQ-Tag, $15 \mathrm{~cm}$ length, $3.9 \mathrm{~mm}$ internal diameter; Waters) and a mobile phase of methanol-100 mm-acetate buffer, pH $6 \cdot 0(30: 70, \mathrm{v} / \mathrm{v}), 1 \mathrm{ml} / \mathrm{min}$ flow rate, $262 \mathrm{~nm}$ wavelength and column oven temperature $40^{\circ} \mathrm{C}$.

\section{Quantitative PCR}

Quantitative PCR was performed to determine PepT-1 mRNA levels in the ileum and colon of NP- and HP-fed piglets at PND7, PN28 and PN160. Analyses were performed as described previously ${ }^{(4,16)}$ using specific primers for PepT-1 (5'-TCCATCAATGACCTCACAGACTTC-3'; 5'-AGCGCCACGTGCACAGA-3'). Transcript concentration was normalised to the transcript concentration of glyceraldehyde 3-phosphate dehydrogenase (Gapdh, reference gene, 5'-CATCCATGACAACTTCGGCA-3'; $5^{\prime}$-GCATGGACTGTGGTCATGAGTC- $3^{\prime}$ ) in the same sample. Gapdh expression was not affected by the tested factors.

\section{Western blot analysis}

Colonic mucosa protein was extracted in a TEX $1 \times(10 \mathrm{~mm}-$ Tris-HCl, $0 \cdot 1$ mm-EDTA, 0.01\% Triton X-100), $60 \mathrm{~mm}$ Trisbase, $\mathrm{pH} 6 \cdot 8,10 \%$ glycerol, 3\% SDS, 5\% $\beta$-mercaptoethanol and a protease inhibitor cocktail (104 mm-4-(2-aminoethyl)benzenesulfonyl fluoride, $80 \mu \mathrm{M}$-aprotinin, 4 mM-bestatin, 1.4 mm-E-64, 2 mm-leupeptin, 1.5 mm-pepstatin A; Sigma-Aldrich) buffer. Levels of Hsp27 and Hsp70 were then determined by Western blot as described previously ${ }^{(22)}$. Densitometry of blots was measured and expressed relatively to $\beta$-actin density of the corresponding sample.

\section{Statistical analysis}

Data were analysed using the program GraphPad Prism (GraphPad Software, Inc.). In Expt 1, a two-way ANOVA was performed, testing piglet age, piglet birth weight status and the interaction between these two factors. Data from Expt 1 and Expt 2 (PND7 and PND28) were combined and analysed by one-way ANOVA. The $t$-test with Bonferroni correction was used as a subsequent multiple comparison test. Data from piglets at PND160 in Expt 2 were analysed using a $t$ test for basal parameters. Colonic barrier function responses to luminal stress were analysed using a twoway ANOVA, testing the neonatal diet, luminal treatment and the interaction between these two factor effects. The $t$-test with Bonferroni correction was used as a subsequent multiple comparison test. Data are presented as means with their standard errors. A $P$ value $\leq 0 \cdot 05$ was considered significant.

\section{Results}

Peptide transporter 1 activity in suckling low-birth-weight and normal-birth-weight piglets during the neonatal period

CFX fluxes across the proximal jejunum mucosa were similar in LBW and NBW piglets, irrespective of their postnatal age (Fig. 1(a)). In the ileum, no difference in CFX flux was observed between the NBW and LBW piglets at PND2. However, LBW piglets exhibited a 5.3-fold greater flux of CFX $(P=0.035$; Fig. 1(b)) at PND7. Flux of CFX across the ileum 

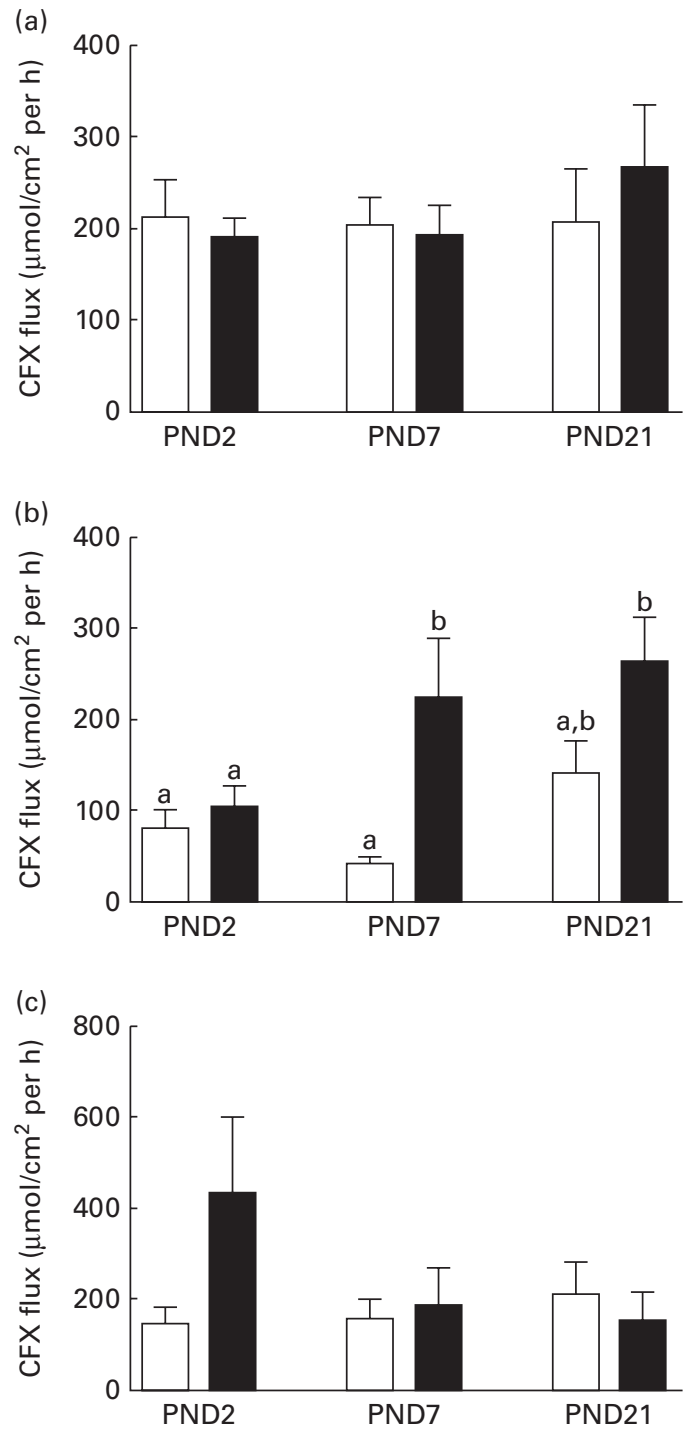

Fig. 1. Flux of cephalexin (CFX) across the different intestinal segments of normal-birth-weight (NBW) and low-birth-weight (LBW) suckling piglets at different postnatal ages. CFX fluxes across the (a) proximal jejunum, (b) ileum and (c) proximal colon were determined at postnatal day (PND) 2, PND7 and PND21 in NBW ( $\square$ ) and LBW ( $\square$ ) piglets. Values are means ( $n$ 5-6 per group), with their standard errors represented by vertical bars. ${ }_{\mathrm{a}, \mathrm{b}}$ Mean values with unlike letters were significantly different $(P<0.05)$.

tended to stay greater in LBW than NBW piglets $(P=0.09$; Fig. 1(b)) at PND21. In the proximal colon, although the CFX flux was increased 3-fold in LBW compared with NBW piglets at PND2, the difference did not reach significance $(P=0 \cdot 17$; Fig. 1(c)). No difference was observed thereafter.

\section{Effect of a high protein formula on peptide transporter 1} activity and barrier function in low birth weight piglet ileum and colon

During the neonatal period. Formula feeding did not change CFX flux across the ileal tissue of LBW piglets at PND7, irrespective of the level of protein in the formula (Fig. 2(a)). At PND28, no difference in ileal CFX flux was observed between the NP- and HP-fed piglets (Fig. 2(a)). In the colon, NP formula feeding did not alter CFX flux across the mucosa compared with suckled LBW piglets. However, feeding a HP formula resulted in greater CFX flux across the colonic mucosa, especially at PND7 where CFX flux was enhanced 3.6-fold in HP- compared with NP-fed piglets (Fig. 2(b)). PepT-1 mRNA relative expression was unchanged by the type of formula in both segments and at both ages (Table 2).

Later in life. At PND160, neither ileal barrier function nor CFX flux across the ileum was different between the NP- and HP-fed pigs (Table 3). Stress conditions ( $\mathrm{pH} 6.0$ buffer or $\mathrm{pH}$ $6 \cdot 0$ buffer + f-MLP) did not modify ileal permeability (data not shown). Basal colonic barrier function parameters as well as CFX flux across the colonic mucosa of NP or HP formulafed piglets were also similar between the NP- and HP-fed pigs (Table 3 ). When luminal $\mathrm{pH}$ was brought to $6 \cdot 0$, colonic flux of FD-4 dramatically increased compared with $\mathrm{pH} 7 \cdot 4$ in the colonic tissue of HP- but not NP-fed pigs (Fig. 3). The addition of $\mathrm{f}-\mathrm{MLP}$ to the mucosal side of the colonic tissue restored FD-4 flux in HP-fed pigs $(P<0 \cdot 001$, Fig. 3). Horseradish peroxidase flux across the colonic mucosa was not significantly altered by acidic $\mathrm{pH}$ or by the addition of f-MLP, irrespective of the pig neonatal diet (data not shown).
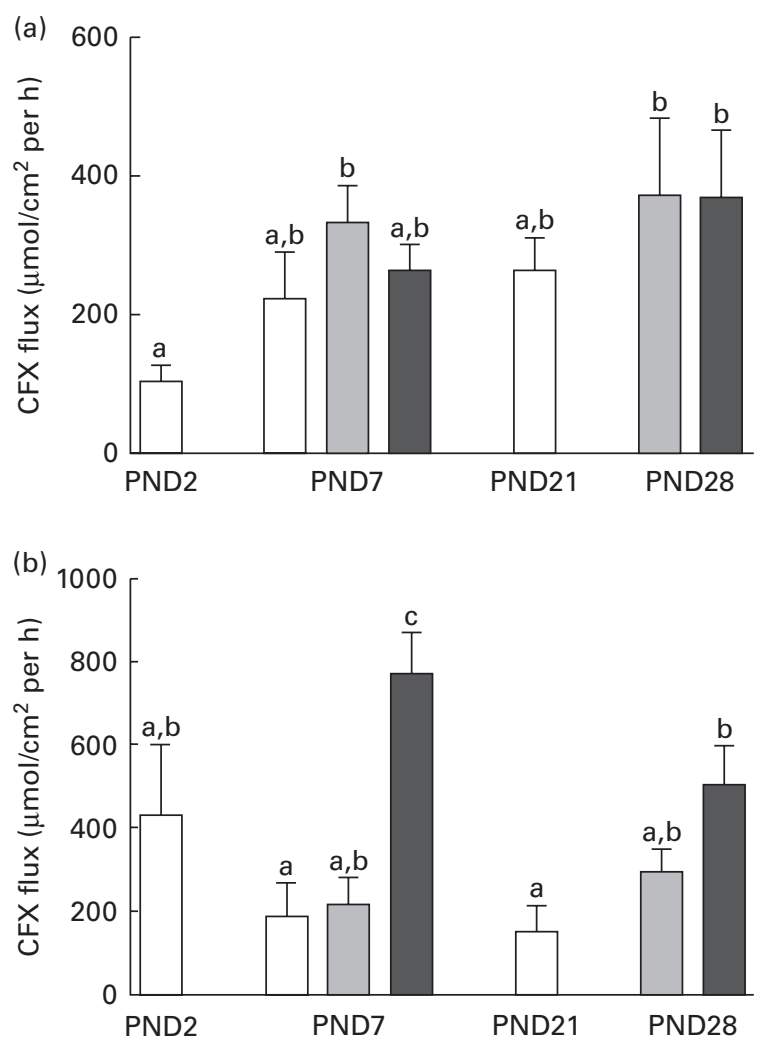

Fig. 2. Flux of cephalexin (CFX) across the ileal and colonic mucosa of lowbirth-weight (LBW) suckled piglets or piglets fed formulas differing in protein content. CFX flux across the (a) ileal and (b) proximal colon was determined at postnatal day (PND) 2, PND7, PND21 and PND28 in LBW suckled piglets $(\square)$ or in LBW piglets fed either a normal-protein $(\square)$ or a high-protein formula ( $\square$ ) from PND2 to PND28. Values are means ( $n$ 5-6 per group), with their standard errors represented by vertical bars. ${ }^{a, b, c}$ Mean values with unlike letters were significantly different $(P<0.05)$. 
Table 2. Ileal and colonic peptide transporter 1 (PepT-1) mRNA levels at postnatal day (PND) 7 and PND28*

(Mean values with their standard errors)

\begin{tabular}{|c|c|c|c|c|c|c|c|c|}
\hline & \multicolumn{4}{|c|}{ PND7 } & \multicolumn{4}{|c|}{ PND28 } \\
\hline & \multicolumn{2}{|c|}{ NP } & \multicolumn{2}{|c|}{$\mathrm{HP}$} & \multicolumn{2}{|c|}{ NP } & \multicolumn{2}{|c|}{$\mathrm{HP}$} \\
\hline & Mean & SEM & Mean & SEM & Mean & SEM & Mean & SEM \\
\hline Ileum & 1.0 & 0.2 & 0.8 & 0.1 & 0.7 & 0.1 & 0.5 & 0.03 \\
\hline Colon & $1 \cdot 1$ & 0.6 & 1.0 & 0.2 & 1.6 & 0.4 & $2 \cdot 2$ & 0.4 \\
\hline
\end{tabular}

$\mathrm{NP}$, normal protein; HP, high protein.

* Levels of PepT-1 mRNA (normalised to that of glyceraldehyde 3-phosphate dehydrogenase (Gapdh)) were measured at PND7 and PND28 in the ileum and colon of low-birth-weight piglets that were fed the NP or HP formula from PND2 to PND28.

To evaluate whether Hsp was involved in the protective effect of f-MLP on barrier function, we measured the levels of Hsp27 and Hsp70 in the colonic mucosa by Western blot at PND160. No difference between the dietary groups was observed (Hsp27: NP 0.42 (SEM 0.08) v. HP 0.41 (SEM 0.07), $P=0.92$ and Hsp70: NP 0.95 (SEM 0.09) $v$. HP 0.93 (SEM 0.10), $P=0 \cdot 88$ ). Interestingly, Hsp27 levels in colonic mucosa correlated negatively with FD-4 fluxes across the colonic mucosa in luminal acidic conditions in HP-fed pigs (Fig. 4(a)). The flux increase from neutral to acidic conditions also correlated negatively with Hsp27 levels, whereas the decrease induced by the addition of f-MLP correlated positively with Hsp27 levels in HP-fed pigs (Fig. 4(b) and (c), respectively). No such correlation was observed in NP-fed animals or with Hsp70 levels (data not shown).

\section{Discussion}

PepT-1 is a singular intestinal transporter exhibiting multifaceted function, from peptide and amino-acid supply to bacterial-host communication controlling gut homeostasis and inflammation. It has also unique developmental features with transient high expression in the colon of neonatal animals during very early life and many regulatory pathways in health and disease. The present study extends this pattern of peculiar regulatory pathways to LBW neonates whose nutrient requirements, intestinal development and gut homeostasis characteristics are overlooked but yet of great importance for neonatal care givers. We demonstrated that LBW neonatal piglets exhibited greater PepT-1 activity in the ileum during the neonatal period compared with NBW piglets. Furthermore, increasing dietary protein supply with HP formula feeding increased PepT-1 activity in the colon. Later in life, colonic barrier function of HP-fed pigs was more sensitive to luminal stress (modelled here by a mild change in luminal $\mathrm{pH}$ ) than that of NP-fed pigs. Furthermore, f-MLP conferred protection to HP colonocyte barrier function. This protective effect of the bacterial peptide was unlikely accounted for a higher entry into colonocytes since PepT-1 activity was similar in the colon of NP- and HP-fed pigs at that age. Conversely, a role for Hsp27 is conceivable as demonstrated by the negative correlations between Hsp27 levels in the colon and the colonic response to stress and the positive correlation between Hsp27 levels and barrier function restoration by f-MLP.

\section{Peptide transporter 1 activity in low-birth-weight piglets}

LBW piglets are characterised by delayed maturation of intestinal functions during the 1st days of life compared with NBW piglets ${ }^{(4,23)}$ followed by catch-up for some but not all intestinal functions, resulting in no apparent gross anatomical difference between LBW and NBW piglets but showing transcriptomic, proteomic and functional differences at the end of the neonatal period or immediately after weaning ${ }^{(24-26)}$. Our data demonstrate increased PepT-1 activity, illustrated with CFX flux, in the ileum of LBW compared with NBW piglets with a significant difference at PND7. One could argue that CFX crosses intestinal mucosa not only through PepT-1 but also through tight junctions in the paracellular space. However, paracellular transport of CFX is unlikely for two reasons. First, we (G Boudry and I Le Huerou-Luron, unpublished results) and others ${ }^{(18)}$ demonstrated that CFX transport is a saturating process, whereas paracellular leak would be non-saturating. Second, we previously observed lower paracellular

Table 3. lleal and colonic barrier function parameters and cephalexin (CFX) fluxes at postnatal day (PND) 160* (Mean values with their standard errors)

\begin{tabular}{|c|c|c|c|c|c|c|c|c|}
\hline & \multicolumn{4}{|c|}{ Ileum } & \multicolumn{4}{|c|}{ Colon } \\
\hline & \multicolumn{2}{|c|}{ NP } & \multicolumn{2}{|c|}{$\mathrm{HP}$} & \multicolumn{2}{|c|}{ NP } & \multicolumn{2}{|c|}{$\mathrm{HP}$} \\
\hline & Mean & SEM & Mean & SEM & Mean & SEM & Mean & SEM \\
\hline$I_{\mathrm{sc}}\left(\mu \mathrm{A} / \mathrm{cm}^{2}\right)$ & 50 & 10 & 85 & 19 & 60 & 21 & 66 & 19 \\
\hline$R\left(\mathrm{mS} . \mathrm{cm}^{2}\right)$ & 21 & 2 & 18 & 3 & 22 & 7 & 12 & 4 \\
\hline FD4 flux $\left(\mathrm{ng} / \mathrm{cm}^{2}\right.$ per $\left.\mathrm{h}\right)$ & 597 & 64 & 518 & 69 & 711 & 96 & 852 & 114 \\
\hline HRP flux (ng/cm² per h) & 40 & 9 & 64 & 17 & 120 & 16 & 95 & 23 \\
\hline CFX flux $\left(\mu \mathrm{mol} / \mathrm{cm}^{2}\right.$ per $\left.\mathrm{h}\right)$ & 205 & 26 & 197 & 30 & 671 & 158 & 652 & 106 \\
\hline
\end{tabular}

$\mathrm{NP}$, normal protein; HP, high protein; $I_{\mathrm{sc}}$, short-circuit current; $R$, transepithelial resistance; FD4, fluorescein isothiocyanatedextran 4000; HRP, horseradish peroxidase.

* Intestinal barrier function parameters $\left(I_{\mathrm{sc}}, R\right.$, flux of FD4 $(4 \mathrm{kDa})$ and HRP $\left.(40 \mathrm{kDa})\right)$ as well as flux of CFX across the ileum and colon mounted in Ussing chambers were measured in PND160 pigs that had been fed the NP or HP formula from PND2 to PND28. 


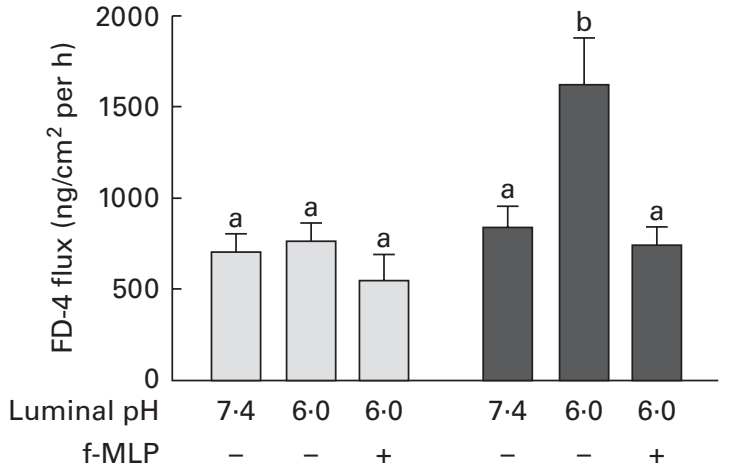

Fig. 3. Flux of fluorescein isothiocyanate-dextran 4000 (FD-4) across the colonic mucosa of adult low-birth-weight (LBW) pigs fed formulas differing in protein content during the neonatal period. Flux of FD-4 across the colonic mucosa of LBW pigs fed a either a normal-protein $(\square)$ or a high-protein formula $(\square)$ from postnatal day (PND) 2 to PND28 followed by a standard pig diet from PND29 to 160 was quantified in Ussing chambers (World Precision Instrument) either in neutral or lightly acidic $\mathrm{pH}$ conditions and with or without $100 \mathrm{~mm}-\mathrm{N}$-formyl methionyl-leucyl-phenylalanine (f-MLP). Values are means ( $n$ 5-6 per group), with their standard errors represented by vertical bars. a,b Mean values with unlike letters were significantly different $(P<0.05)$.

permeability in the ileum of LBW compared with NBW piglets at PND28 ${ }^{(27)}$, which would not fit with enhanced CFX flux if it was a paracellular passage.

Our previous data showed that the delay in PepT-1 mRNA maturation was transient in early life and that by PND5, Pept-1 mRNA levels were similar in NBW and LBW piglets in the ileum and colon ${ }^{(4)}$. Functionally, this did not translate into enhanced PepT-1 activity at PND2 but a few days later (PND7) in the ileum with no significant difference in PepT-1 activity in the colon. Discrepancy between mRNA levels, protein expression and activity of PepT-1, especially in the colon, has already been observed either in healthy or diseased states $^{(28)}$. A very recent report has demonstrated increased PepT-1 mRNA but not protein expression in LBW rat pups born to dams fed a low-protein diet compared with NBW pups $^{(29)}$, corroborating differences between LBW and NBW neonates at the gene expression level that do not translate into difference in function.

\section{Effect of high-protein formula during the neonatal period}

Several studies have demonstrated that HP or high peptide intake increases PepT-1 expression in vitro in intestinal epithelial cell lines ${ }^{(30,31)}$ or in vivo in the small intestine of adult rodents ${ }^{(32,33)}$. PepT-1 regulation by its own substrate during the neonatal period has not been reported yet. In our case, the HP formula, which provided $40 \%$ more protein than the $\mathrm{NP}$ one, failed to induce any change in small-intestinal PepT-1 activity but increased the activity in the colon, especially at PND7. Formula feeding per se did not appear to alter PepT-1 activity when protein content was matching that of sow milk as demonstrated by similar ileal and colonic CFX fluxes between NP formula-fed and suckled piglets. We previously observed that protein level in the distal ileum was increased (25\%) at PND7 but not at PND28 in HP-compared with NP-fed piglets ${ }^{(17)}$, suggesting that protein concentration
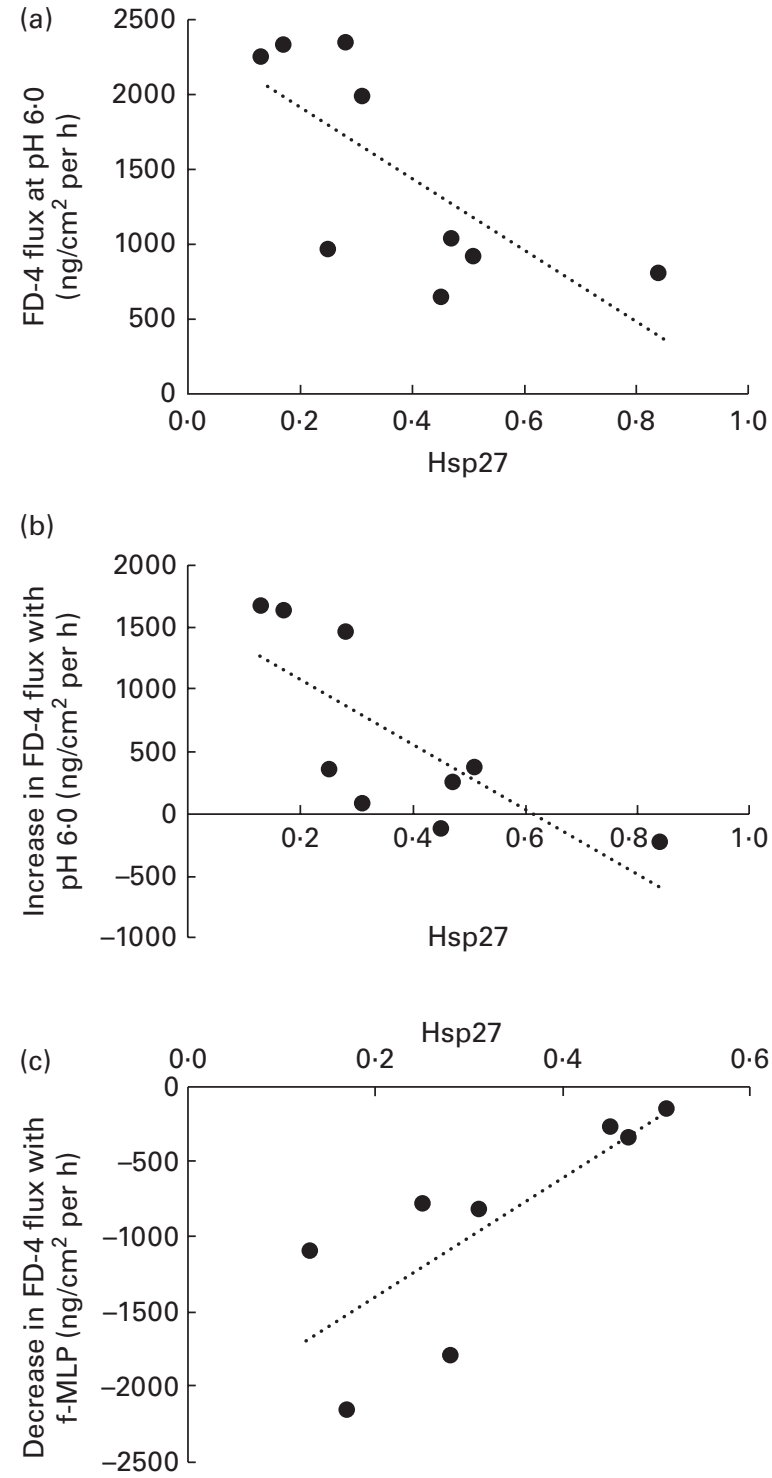

Fig. 4. Correlation between heat shock protein 27 (Hsp27) in the colonic mucosa of high protein-fed pigs and change in fluorescein isothiocyanatedextran 4000 (FD-4) flux of colonic segments subjected to different conditions in Ussing chambers (World Precision Instrument). Level of Hsp27 quantified by Western blot was correlated to FD-4 flux measured in slightly acidic conditions (a) or the difference in FD-4 flux between $\mathrm{pH} 7.0$ and $\mathrm{pH} 6.0$ conditions (b) or between tissues kept at $\mathrm{pH} 6.0$ with or without the addition of f-MLP on the luminal side (c). (a) $r-0.714, P=0.03$; (b) $r-0.747$, $P=0.02$ and (c) $r 0.778, P=0.02$.

in the luminal content was greater in our HP-fed piglets at least during the 1st week of HP formula feeding. The reason why PepT-1 activity was not enhanced in the small intestine by the increased substrate availability is unclear. It can be speculated that PepT-1 expression and activity in the small intestine are maximal during the neonatal period as illustrated by the spectacular fall in PepT-1 mRNA level between 3-week-old unweaned and 6-week-old fully weaned Yucatan piglets ${ }^{(5)}$ and cannot be further enhanced by dietary protein. In the colon, PepT-1 regulation by protein might be more flexible and the $25 \%$ increase of protein 
flowing through the ileo-caecal junction might be sufficient to enhance PepT-1 activity. Another possible pathway for specific up-regulation of PepT-1 activity in the colon could be pro-inflammatory signals since PepT-1 expression and activity have been shown to be induced by TNF- $\alpha$ and interferon- $\gamma^{(34)}$. However, our previous data did not show any sign of inflammation in the colon of piglets fed a HP formula $^{(17)}$, weakening this hypothesis.

\section{Effect of the high-protein formula on colonic barrier function later in life}

The present study provides evidence that feeding a HP formula during the neonatal life has long-term consequences on colonic homeostasis. We show that a mild stress (slightly acidic $\mathrm{pH}$ ) alters barrier function in the colonic mucosa of $\mathrm{HP}$ - but not NP-fed pigs. In Caco- $2_{\mathrm{BBe}}$ cells, a decrease in $\mathrm{pH}$ from $7 \cdot 4$ down to $6 \cdot 0$ does not modify epithelial permeability, whereas a $\mathrm{pH}$ of 5.5 dramatically increases monolayer permeability, suggesting that intestinal epithelial cells are able to maintain epithelial barrier integrity up to a certain level of metabolic stress ${ }^{(20)}$. Our data suggest that neonatal HP feeding alters the sensitivity of intestinal epithelial cells to metabolic stress in adulthood. The effect of $\mathrm{pH}$ change was rapid since a change in permeability was observed within the $2 \mathrm{~h}$ of the Ussing chamber experiment. Colonic barrier function in basal conditions was not different between the NP- and HP-fed pigs, suggesting that expression of tight junction proteins was similar between the two groups. The change in colonic permeability with the acidic $\mathrm{pH}$ was therefore either linked to the change in tight junction localisation or phosphorylation and cytoskeleton reorganisation as already established with other metabolic stresses ${ }^{(35)}$, or to altered metabolic rescue pathways required under metabolic stress. These data are in line with our previous data showing that female colonic mucosa of pigs fed a HP formula during early life was more sensitive to oxidative stress or inflammatory mediators than the colonic mucosa of NP-fed pigs ${ }^{(17)}$.

Interestingly, addition of f-MLP, a pro-inflammatory peptide transported by PepT-1, reduced the colonic hyper-permeability observed with acidic conditions in HP-fed animals. Previous reports showed that pretreatment with f-MLP protected intestinal epithelial cells from oxidant- or inflammatory mediator-induced depolymerisation of actin and a decrease in transepithelial resistance. This effect was probably mediated by the induction of Hsp27 into epithelial cells since silencing Hsp27 expression inhibited the protective effect of $\mathrm{f}-\mathrm{MLP}^{(14)}$. A similar Hsp27-driven protective effect by f-MLP is plausible in the present study since Hsp27 levels correlated negatively with the magnitude of response to the acidic challenge and positively with the magnitude of response to f-MLP. However, we must stress out that Hsp27 measured here is constitutive Hsp27 and not Hsp27 induced by the acidic challenge or f-MLP incubation. Moreover, these correlations were observed only in HP- not NP-fed animals that displayed no reaction to the acidic challenge. Finally, the mean level of Hsp27 in colonic mucosa was not different between the NP- and HP-fed pigs. A complex relationship between priming of epithelial cells during the neonatal period induced by a greater invasion of bacterial peptides into colonocytes through PepT-1 and response to f-MLP and induction of Hsp27 later in life in HP-fed pigs can therefore be hypothesised.

Taken together, these experiments demonstrate that LBW neonates have unique intestinal peptide transport capacity that can be greatly influenced by the diet, especially in the colon. The nutritional management of LBW infants that are routinely fed a HP formula may result in enhanced activity of the transporter in the colon during early life. This practice seems also to have consequences on colonic homeostasis later in life. Considering the high level of potentially harmful bacterial peptides present in that part of the gastrointestinal tract at both periods of life, immunological consequences and mechanisms warrant further investigations.

\section{Acknowledgements}

The authors would like to thank all the individuals involved in animal care. They also acknowledge the help of Soraya Benghabrit and Nadine Mezière.

The present study was supported by the Agence Nationale de la Recherche, PROTNEONAT- ANR-05-PNRA-09.

The authors' contributions are as follows: G. B. and I. L. H.-L. formulated the research question; G. B., A. J., G. S. and I. L. H.-L. designed the study; G. B., V. R., C. P., A. J., G. S. and I. L. H.-L. carried out the study; G. B., V. R., C. P. and G. S. analysed the data; G. B. and I. L. H.-L. wrote the paper.

The authors declare that they have no conflict of interest.

\section{References}

1. Boudry G, David ES, Douard V, et al. (2010) Role of intestinal transporters in neonatal nutrition: carbohydrates, proteins, lipids, minerals, and vitamins. I Pediatr Gastroenterol Nutr 51, 380-401.

2. Shen H, Smith DE \& Brosius FC (2001) Developmental expression of PEPT1 and PEPT2 in rat small intestine, colon and kidney. Pediatr Res 49, 789-795.

3. Gilbert ER, Wong EA \& Webb KE (2008) Peptide absorption and utilization: implications for animal nutrition and health. J Anim Sci 86, 2135-2155.

4. D'Inca R, Gras-Le Guen C, Che L, et al. (2011) Intrauterine growth restriction delays feeding-induced gut adaptation in term newborn pigs. Neonatology 99, 208-216.

5. Nosworthy MG, Bertolo RF \& Brunton JA (2013) Ontogeny of dipeptide uptake and peptide transporter 1 (PepT1) expression along the gastrointestinal tract in the neonatal Yucatan miniature pig. BrJ Nutr 110, 275-281.

6. Adibi SA (2003) Regulation of expression of the intestinal oligopeptide transporter (Pept-1) in health and disease. $\mathrm{Am}$ J Physiol 285, G779-G788.

7. Thureen P \& Heird WC (2005) Protein and energy requirements of the preterm/low birthweight (LBW) infant. Pediatr Res 57, 95R-98R.

8. Singhal A, Kennedy K, Lanigan J, et al. (2010) Nutrition in infancy and long-term risk of obesity: evidence from 2 randomized controlled trials. Am J Clin Nutr 92, 1133-1144.

9. Charrier L \& Merlin D (2006) The oligopeptide transporter hPepT1: gateway to the innate immune response. Lab Invest 86, 538-546. 
10. Merlin D, Si-Tahar M, Sitaraman SV, et al. (2001) Colonic epithelial hPepT1 expression occurs in inflammatory bowel disease: transport of bacterial peptides influences expression of MHC class 1 molecules. Gastroenterology 120, 1666-1679.

11. Buyse M, Tsocas A, Walker F, et al. (2002) PepT1-mediated fMLP transport induces intestinal inflammation in vivo. Am J Physiol 283, C1795-C1800.

12. von Ritter C, Sekizuka E, Grisham MB, et al. (1988) The chemotactic peptide $N$-formyl methionyl-leucyl-phenylalanine increases mucosal permeability in the distal ileum of the rat. Gastroenterology 95, 651-656.

13. Chen K, Liu M, Liu Y, et al. (2013) Formylpeptide receptor-2 contributes to colonic epithelial homeostasis, inflammation, and tumorigenesis. J Clin Invest 123, 1694-1704.

14. Carlson RM, Vavricka SR, Eloranta JJ, et al. (2007) fMLP induces Hsp27 expression, attenuates NF-кB activation, and confers intestinal epithelial cell protection. Am J Physiol 292, G1070-G1078.

15. Barreau F, Cartier C, Ferrier L, et al. (2004) Nerve growth factor mediates alterations of colonic sensitivity and mucosal barrier induced by neonatal stress in rats. Gastroenterology 127, 524-534.

16. Chatelais L, Jamin A, Gras-Le Guen C, et al. (2011) The level of protein in milk formula modifies ileal sensitivity to LPS later in life in a piglet model. PLOS ONE 6, e19594.

17. Boudry G, Jamin A, Chatelais L, et al. (2013) Dietary protein excess during neonatal life alters colonic microbiota and mucosal response to inflammatory mediators later in life in female pigs. J Nutr 143, 1225-1232.

18. Luckner P \& Brandsch M (2005) Interaction of $31 \beta$-lactam antibiotics with the $\mathrm{H}^{+}$/peptide symporter PEPT2: analysis of affinity constant and comparison with PEPT1. Eur J Pharm Biopharm 59, 17-24.

19. Sullivan HR, Billings RE \& McMahon RE (1969) Metabolism of cephalexin-14C in mice and in rats. I Antibiot 22 $195-200$

20. Unno N, Menconi MJ, Smith MA, et al. (1997) Acidic conditions ameliorate both adenosine triphosphate depletion and the development of hyperpermeability in cultured Caco- 2 BBe enterocyte monolayers subjected to metabolic inhibition. Surgery 121, 668-680.

21. Unno N, Hodin RA \& Fink MP (1999) Acidic conditions exacerbate interferon-gamma-induced intestinal epithelial hyperpermeability: role of peroxynitrous acid. Crit Care Med 27, 1429-1436.

22. Lallès JP \& David JC (2011) Fasting and refeeding modulate the expression of stress proteins along the gastrointestinal tract of weaned pigs. J Anim Physiol Anim Nutr (Berl) 95 , $478-488$

23. D'Inca R, Kloareg M, Gras-Le Guen C, et al. (2010) Intrauterine growth restriction modifies the developmental pattern of intestinal structure, transcriptomic profile, and bacterial colonization in neonatal pigs. J Nutr 140, 925-931.
24. Wang $\mathrm{X}$, Wu W, Lin G, et al. (2010) Temporal proteomic analysis reveals continuous impairment of intestinal development in neonatal piglets with intrauterine growth restriction. J Proteome Res 9, 924-935.

25. Michiels J, De Vos M, Missotten J, et al. (2013) Maturation of digestive function is retarded and plasma antioxidant capacity lowered in fully weaned low birth weight piglets. Br J Nutr 109, 65-75.

26. D'inca R (2010) Modulation nutritionnelle du développement post-natal de l'intestin chez le porcelet: restriction nutritionnelle in-utero et al imentation hyperprotéique (Nutritional modulation of intestinal postnatal development in piglets: in utero growth restriction and high-protein diet). Thèse de l'Université de Rennes 1, Rennes, 194p.

27. Boudry G, Morise A, Seve B, et al. (2011) Effect of milk formula protein content on intestinal barrier function in a porcine model of LBW neonates. Pediatr Res 69, 4-9.

28. Radeva G, Buyse M, Hindlet P, et al. (2007) Regulation of the oligopeptide transporter, PEPT-1, in DSS-induced rat colitis. Dig Dis Sci 52, 1653-1661.

29. Pinheiro DF, Pinheiro PF, Buratini J Jr, et al. (2013) Maternal protein restriction during pregnancy affects gene expression and immunolocalization of intestinal nutrient transporters in rats. Clin Sci (Lond) 125, 281-289.

30. Thamotharan M, Bawani SZ, Zhou X, et al. (1998) Mechanism of dipeptide stimulation of its own transport in a human intestinal cell line. Proc Assoc Am Physicians 110, 361-368.

31. Walker D, Thwaites DT, Simmons NL, et al. (1998) Substrate upregulation of the human small intestinal peptide transporter, hPepT1. J Physiol 507, 697-706.

32. Erickson RH, Gum JR Jr, Lindstrom MM, et al. (1995) Regional expression and dietary regulation of rat small intestinal peptide and amino acid transporter mRNAs. Biochem Biophys Res Commun 216, 249-257.

33. Shiraga T, Miyamoto KI, Tanaka H, et al. (1999) Cellular and molecular mechanisms of dietary regulation on rat intestinal $\mathrm{H}^{+} /$peptide transporter PepT1. Gastroenterology $\mathbf{1 1 6}$, $354-362$.

34. Vavricka SR, Musch MW, Fujiya M, et al. (2006) Tumor necrosis factor-alpha and interferon-gamma increase PepT1 expression and activity in the human colon carcinoma cell line Caco-2/bbe and in mouse intestine. Pflugers Arch 452, $71-80$.

35. Nazli A, Yang PC, Jury J, et al. (2004) Epithelia under metabolic stress perceive commensal bacteria as a threat. Am J Pathol 164, 947-957.

36. Dourmad JY, Noblet J \& Etienne M (1998) Effect of protein and lysine supply on performance, nitrogen balance, and body composition changes of sows during lactation. J Anim Sci 76, 542-550. 